\begin{tabular}{llll}
\multicolumn{2}{l}{ Abstract P059 Table 1 } & & \\
\hline & 2019 & 2020 & $\begin{array}{l}\text { P } \\
\text { VALUE }\end{array}$ \\
\hline Number of referrals & 62 & 37 & \\
Mean age & 69 & 69 & \\
Sex (M:F) & $53(85.5 \%): 9$ & $30(81.1 \%): 7$ & \\
& $(14.5 \%)$ & $(18.9 \%)$ & 0.39 \\
Mean size largest lesion (cm) & 5.8 & 6.1 & 0.48 \\
Mean number of lesions & 1.96 & 1.53 & 0.08 \\
Mean time to MDT discussion & 20.8 & 11.2 & \\
(days) & & & 0.03 \\
Mean time to first treatment & 93.2 & 53.8 & \\
(days) & & & \\
\hline
\end{tabular}

number of diagnoses in the 2020 cohort compared with the 2019 cohort. The mean time from diagnosis to discussion at the MDT meetings was reduced in the 2020 cohort (20.8 vs 11.2 days) and the mean time to first treatment was also reduced (93.2 vs 53.8 days).

There was no evidence of patients presenting at a more advanced stage in 2020. Data used in the BCLC staging criteria was used as a marker of disease progression. There was no significant difference in the size of lesions $(p=0.39)$ or the number of lesions $(p=0.94)$ in the 2 cohorts.

Conclusion During the COVID-19 pandemic there was a $40 \%$ reduction in HCC diagnoses referred to the WOS MDT, compared with 2019. However there is no evidence that patients have presented with more advanced disease. Additionally, we have demonstrated a reduction in the referral to treatment time where active treatment was indicated. Given that the incidence of chronic liver disease has continued to rise in our population, we would expect an increased number of patients to present with more advance stage HCC over the coming months.

\section{P060 RITUXIMAB AS THIRD LINE THERAPY IN IGG4-RELATED DISEASE: EXPERIENCE FROM A MULTI-CENTRE COHORT}

${ }^{1}$ Charis D Manganis*, 'Chen X Lee, ${ }^{1}$ Rory JR Peters, ${ }^{1}$ Joel David, ${ }^{1}$ Raashid Luqmani, ${ }^{2}$ Sinisa Savic, ${ }^{3}$ William Spiller, ${ }^{4}$ Eoghan McCarthy, ${ }^{2}$ Matthew Huggett, ${ }^{4}$ Joe Geraghty, ${ }^{5}$ Manu Nayar, ${ }^{5}$ Josie Vila, ${ }^{1}$ Emma L Culver. ' Oxford University Hospitals NHS Foundation Trust, UK; ${ }^{2}$ Leeds Teaching Hospitals NHS Trust, UK; ${ }^{3}$ Salford Royal NHS Foundation Trust, UK; ${ }^{4}$ Manchester University NHS Foundation Trust, UK; ${ }^{5}$ The Newcastle upon Tyne Hospitals NHS Foundation Trust, UK

\subsection{6/gutjnl-2021-BASL.69}

Introduction Immunoglobulin G4-Related Disease (IgG4RD) is a rare multi-organ fibro-inflammatory immune-mediated disorder that has a predilection for the pancreas and bile ducts, leading to organ damage and failure, and an increased risk of malignancy. B cells play a crucial role in disease pathogenesis and disease relapse. Rituximab (anti-CD20 chimeric monoclonal antibody) has received UK NICE approval for third-line therapy for intolerance/relapse on glucocorticoids and conventional immunomodulators. We sought to assess the indications, clinical response and outcome to Rituximab in a multi-centre UK cohort of IgG4RD patients.
Methods Data was retrospectively collected from four UK tertiary referral centres for IgG4RD patients that received Rituximab therapy, including baseline demographics, clinical characteristics, disease phenotype, indication for rituximab, dosing schedule, preceding treatment, outcomes, and complications. Wilcoxon paired test was used for numerical and chisquared test for categorical variables.

Results Forty-three patients received rituximab for IgG4RD; $30(70 \%)$ were male, median age at diagnosis was 60 years (range 19-79). Thirty-three patients (77\%) had raised serum IgG4 at presentation, thirty-two patients (74\%) had multiorgan disease. Disease phenotypes were hepatopancreatobiliary (21\%), head and neck (30\%), retroperitoneal and aorta (5\%) and systemic disease (44\%).

The median age at time of rituximab infusion was 65 years (range 20-82), and median duration between diagnosis and rituximab was 38 months (range 1 to 148). Indications for rituximab included disease refractory to steroids and/or other immunosuppressants (49\%), relapse following remission (42\%) and intolerance to steroids and/or immunosuppressants (7\%). Treatment preceding rituximab included prednisolone $(35 ; 81 \%)$, azathioprine $(18 ; 42 \%)$, mycophenolate $(15 ; 35 \%)$, methotrexate $(10 ; 23 \%)$ and cyclophosphamide $(9 ; 21 \%)$. Thirty-one patients (72\%) were on dual immunosuppression prior to rituximab.

Clinical response to rituximab was present in all patients with follow-up available $(n=34)$. Serum IgG4 levels fell with rituximab therapy (pre-treatment median $4.99 \mathrm{~g} / \mathrm{l}$, post-treatment median 3.33g/l; p<0.001) (figure 1). Average follow-up was 54 months (range 2 to 156). The median number of cycles received was 1 (range 1-8). The majority had ondemand infusions (77\%) and $10(23 \%)$ received maintenance therapy. Twenty-one patients (62\%) were able to discontinue one or more immunosuppressants/corticosteroids. Six patients had hypogammaglobulinaemia, four of whom were on 6monthly rituximab; none required rescue IVIg. Two patients developed an infection. None developed de novo cardiovascular or malignant complications.

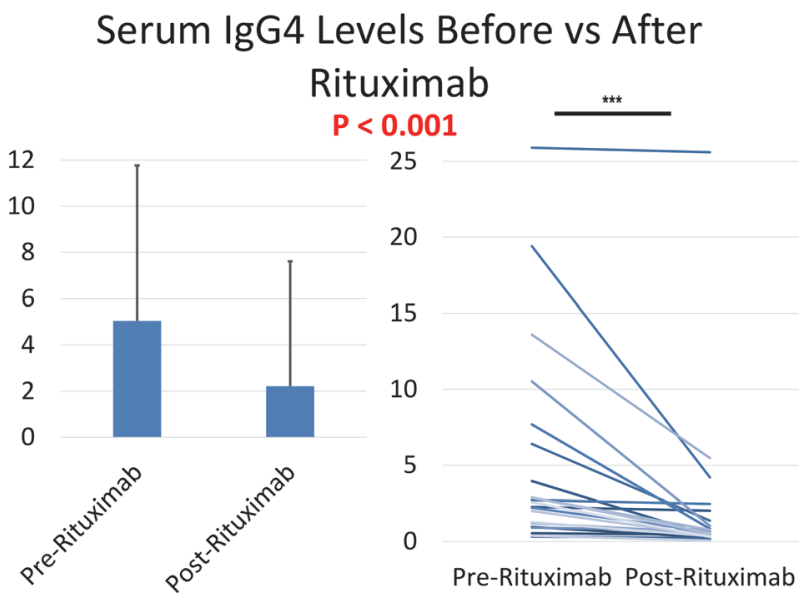

Abstract P060 Figure 1

Conclusion Rituximab was safe and effective as third-line therapy for disease relapse in IgG4RD in this multi-centre prospective UK cohort, allowing discontinuation of steroid/ conventional immunosuppression in the majority. 